\title{
Le développement territorial durable aux échelles infranationales : Le cas du programme de formation médicale à Saguenay
}

\author{
Gilles Bergeron, professeur \\ Université du Québec à Chicoutimi
}

\section{INTRODUCTION}

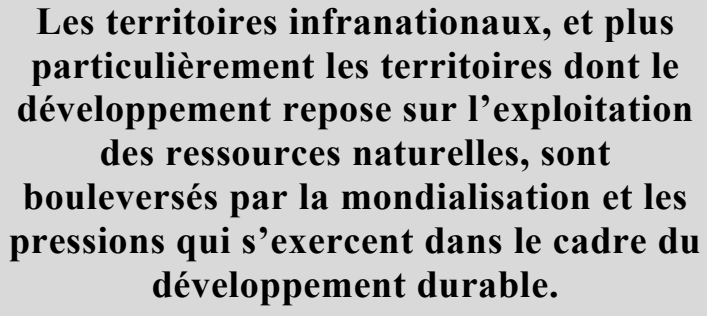

Les territoires infranationaux, et plus particulièrement les territoires dont le développement repose sur l'exploitation des ressources naturelles, sont bouleversés par la mondialisation et les pressions qui s'exercent dans le cadre du développement durable.

Les territoires infranationaux, et plus particulièrement les territoires dont le développement repose sur l'exploitation des ressources naturelles, sont bouleversés par la mondialisation et les pressions qui s'exercent dans le cadre du développement durable. Ces territoires sont menacés d'une déstructuration durable compte tenu des mécanismes cumulatifs du sousdéveloppement qui se mettent en place. L'exploitation des ressources dans le cadre d'une modernisation rapide des entreprises crée de moins en moins d'emplois, ce qui est une source de sous performance économique du territoire. Cette sous-performance économique a un impact négatif sur le marché du travail. Elle provoque le départ d'une partie importante de la population, souvent les plus jeunes et les plus dynamiques, ce qui est une source de sous performance démographique. Cette sous-performance démographique affaiblit à son tour le potentiel de création d'emplois du marché local, l'attractivité et la capacité d'innovation du territoire ce qui est une nouvelle source de sous performance économique.

Les réflexions sur la mise en œuvre du développement territorial durable et sur les territoires favorables à son implantation apportent une contribution nécessaire pour le développement de ces territoires ${ }^{1}$. Nous allons chercher à enrichir cette approche à l'aide d'une étude de cas : l'implantation du PFMS dans le territoire du Saguenay-Lac-Saint-Jean, Québec.

Dans une première partie, nous ferons une présentation du Programme de formation médicale à Saguenay (PFMS) et de la méthodologie que nous avons utilisée pour l'analyse des impacts économiques, sociaux et environnementaux du projet ${ }^{2}$. Une présentation du territoire d'accueil, le Saguenay-Lac-Saint-Jean, et de sa dynamique de développement suivra ${ }^{3}$.

Dans une deuxième partie, nous chercherons à démontrer comment le PFMS permet de contribuer à l'émergence d'une stratégie de développement territorial durable. En tenant compte des écrits théoriques sur cette question ${ }^{4}$, nous analyserons la contribution du projet selon quatre dimensions : sa contribution à l'amélioration de l'efficacité économique, l'amélioration de l'équité sociale, le développement des capacités de mobilisation des acteurs et de la gouvernance, le maintien de l'intégrité de l'environnement et du patrimoine collectif.

En conclusion, à partir de notre étude de cas, nous dégagerons quelques pistes de réflexion sur le concept et la mise en œuvre d'une stratégie de développement territorial durable.

L'exploitation des ressources dans le cadre d'une modernisation rapide des entreprises crée de moins en moins d'emplois, ce qui est une source de sous performance économique du territoire. 


\section{PREMIÈRE PARTIE}

\subsection{Le programme}

\section{La formation médicale offerte est d'une durée de quatre ans et elle est très semblable à celles offertes à Sherbrooke et Moncton : mêmes contenus, même approche pédagogique (apprentissage par problème ou APP), mêmes formations pratiques, mêmes objectifs.}

Depuis le début des années 2000, on a vu se développer dans le monde plusieurs sites de formation médicale situés à distance des campus principaux. On en compte plus d'une centaine, dont la très grande majorité est dédiée à la formation clinique. Il s'agit d'une évolution importante de la formation médicale qui a pris forme dans un contexte d'augmentation des admissions, afin de contrer les pénuries réelles et attendues de ce domaine d'activité ainsi que les problèmes de répartition géographique et disciplinaire des professionnels. On peut y voir un effort réel des universités pour mieux répondre aux besoins des individus et des communautés.

Un très petit nombre (une quinzaine) de sites distants des campus principaux offrent toute la formation dite prédoctorale, conduisant à l'obtention d'un diplôme de médecine. C'est le cas du site de Saguenay où la Faculté de médecine et des sciences de la santé (FMSS) de l'Université de Sherbrooke rend accessible son programme, avec la collaboration du Centre de santé et de services sociaux de Chicoutimi (CSSSC) et de l'Université du Québec à Chicoutimi (UQAC). Ce site est connu sous l'appellation de Programme de formation médicale à Saguenay (PFMS). Il a été implanté simultanément et en symbiose avec un autre site distant à Moncton au Nouveau-Brunswick.

La formation médicale offerte est d'une durée de quatre ans et elle est très semblable à celles offertes à Sherbrooke et Moncton : mêmes contenus, même approche pédagogique (apprentissage par problème ou APP), mêmes formations pratiques, mêmes objectifs. Les stages d'externat commencent en janvier de la troisième année, ils sont presque tous offerts dans la région $\mathrm{du}$
Saguenay-Lac-Saint-Jean, mais les étudiants ont également accès à l'ensemble des milieux cliniques accrédités de la FMSS de l'Université de Sherbrooke.

Le rythme de croisière du PFMS sera de 128 étudiants, à raison de 32 nouvelles admissions par année. C'est seulement depuis 2010 qu'il admet 32 étudiants. Au moment de sa création en 2006, il recevait 24 étudiants.

Une grande distance sépare le PFMS du campus principal de l'Université de Sherbrooke puisque Saguenay est à environ $450 \mathrm{~km}$ de Sherbrooke.

Malgré de très grandes similitudes, le PFMS présente des particularités et développe progressivement sa propre personnalité. Par exemple, il profite de la grande proximité des deux partenaires régionaux (UQAC et CSSC), dont les terrains sont pratiquement adjacents, pour offrir dans des locaux du milieu universitaire toute la partie académique de la formation, et dans des locaux de l'hôpital toute la partie clinique de la formation. Ce qui permet aux étudiants de vivre et d'apprendre sur un campus universitaire complet, tout en acquérant leurs habiletés cliniques (dès le début) dans leur futur milieu de vie professionnelle. On pourrait ajouter que les professeurs sont de la région du Saguenay-LacSaint-Jean et que les médecins de famille constituent une plus grande proportion du corps professoral. De même, le contexte d'une région d'environ 300000 habitants, avec un territoire urbain et rural, est susceptible de contribuer à former graduellement la personnalité du PFMS.

À Saguenay, on a promu quatre cohortes, pour un total de 86 étudiants. L'expérience est encore très jeune, mais on commence à observer certains résultats.

\subsection{La méthodologie}

Les impacts économiques du programme et des activités liées au programme ont été mesurés selon la méthodologie habituelle des études d'impact et avec une analyse rigoureuse des informations recueillies. 
Les revenus et les emplois directs sont ceux du personnel directement impliqué dans le programme et les activités liées au programme; les revenus et les emplois indirects sont ceux liés aux achats de biens et services; les revenus et emplois induits sont ceux liés aux dépenses qui sont réalisées dans le territoire par les deux premiers groupes.

L'année de référence utilisée pour le calcul des revenus et des emplois a été 2011-2012. Les calculs ont été effectués en tenant compte du fait que le programme à maturité comprendra 4 cohortes de 32 étudiants chacune, pour un total de 128 étudiants.

Il est aussi utile de préciser que, dans le cadre de cette étude, ce n'est pas l'impact économique total qui nous intéresse, mais l'impact économique pour les travailleurs du Saguenay-Lac-Saint-Jean. Nous ne prenons en compte que les dépenses qui sont réalisées dans la région et qui donnent lieu à des flux de revenus supplémentaires pour la région.

L'impact est mesuré non en termes de dépenses, mais en termes de revenus et d'emplois pour les travailleurs de la région, des variables plus significatives pour le développement du territoire.

Les impacts sociaux et environnementaux ont été mesurés avec des entrevues qualitatives semistructurées conduites face à face et réalisées entre le 27 mai et le 12 août 2013 auprès de 36 informateurs clés : dont 7 du milieu universitaire, treize du milieu de la santé et seize de la communauté.

La participation à l'enquête était sur une base volontaire et confidentielle. Un formulaire de consentement, lu et signé par les participants, a été préparé en suivant les recommandations du guide du comité d'éthique de la recherche de l'UQAC. Les informations ont été conservées et traitées de façon à assurer l'anonymat des répondants.

Nous avons utilisé le questionnaire préparé par le Centre for Rural and Northern Health Research Lakehead University and Laurentian University ${ }^{5}$, un groupe de recherche qui a fait une étude similaire en Ontario.

Nous avons traduit ce questionnaire et ajouté deux questions pour explorer une amélioration possible des interactions entre le programme et la communauté. Les questions de l'entrevue étaient de type " ouverte » pour permettre aux informateurs clés d'explorer la nature et l'étendue des impacts du programme.

Avec la permission des répondants, les entrevues ont été dactylographiées et analysées en utilisant le programme d'analyse qualitative Nvivo. Les données ont été regroupées par thème en vue de dégager ceux qui faisaient l'objet d'un large consensus sans toutefois négliger les impacts particuliers identifiés par des participants qui avaient une connaissance plus spécifique des impacts du programme.

\subsection{Le territoire d'accueil du projet}

\section{En plus d'être le résultat d'un découpage administratif, le Saguenay-Lac-St-Jean répond aux critères pour se définir comme un territoire de développement durable compte tenu de son histoire, de ses ressources, de ses institutions et de son patrimoine collectif.}

Le Saguenay-Lac-Saint-Jean est la troisième plus grande région du Québec avec une superficie de 95895 kilomètres carrés. Avec une population de 279863 habitants en 2014 répartie principalement autour du Lac-St-Jean et le long de la rivière Saguenay, la densité d'occupation du territoire est faible avec 2,9 habitants au kilomètre carré. Située sur la rive nord du St-Laurent, elle est au centre géographique du Québec, mais occupe une position excentrique par rapport aux grandes villes du Québec et au continent américain.

Le Saguenay-Lac-St-Jean est l'une des dix-sept régions administratives du Québec, elle est composée de quatre municipalités régionales de comté (M.R.C.) et de quarante-neuf municipalités locales.

La Ville de Saguenay, qui est aussi une MRC, représente à elle seule plus de $50 \%$ de la population de la région.

En plus d'être le résultat d'un découpage administratif, le Saguenay-Lac-St-Jean répond aux critères pour se définir comme un territoire de développement durable compte tenu de son histoire, de ses ressources, de ses institutions et de son patrimoine collectif. Il recouvre trois 
différentes dimensions complémentaires : une dimension identitaire, une dimension matérielle et une dimension organisationnelle ${ }^{6}$.

Même si les limites du territoire ont été modifiées légèrement au cours du temps, le Saguenay-LacSaint-Jean est doté d'une identité propre, compte tenu de son éloignement géographique des grands centres, de son histoire commune et de son homogénéité culturelle.

Les deux principales ressources qui sont à la base de son développement, la forêt et l'eau sont dispersées sur l'ensemble du territoire et leur exploitation implique une coordination des activités sur l'ensemble du territoire. Ce sont d'ailleurs les deux mêmes grandes entreprises qui possèdent la très grande partie des usines sur le territoire, ce qui favorise une intégration plus forte. Les infrastructures privées et publiques (aéroport, port de mer, chemin de fer, routes) qui ont été construites répondent à des besoins complémentaires. Il en est de même pour les institutions spécialisées dans le domaine de la santé, de la formation et de la culture.

Les citoyens partagent les mêmes défis collectifs de développement, même si certains enjeux locaux se présentent différemment pour les milieux ruraux et les milieux urbains. Les débats qui animent la communauté régionale font parfois ressortir des différences de point de vue et d'intérêts, mais une communauté d'intérêts plus large permet de mobiliser la collectivité sur des enjeux communs prioritaires. Les démarches de planification territoriale mise en place depuis 1982 pour mobiliser et organiser la collaboration des acteurs en sont une bonne illustration.

En 2013, avec une population de 278069 habitants, le Saguenay-Lac-Saint-Jean regroupait $3,4 \%$ de la population et 3,1\% des emplois du Québec. La population rurale représentait 24,6\% de la population de la région. À titre comparatif, la population rurale du Québec correspondait à $19,3 \%$ de la population de la province. En 2012, le revenu disponible par habitant du SaguenayLac-Saint-Jean est demeuré inférieur à la moyenne québécoise. Il se situait à $25106 \$$, comparativement à $26347 \$$ pour l'ensemble du Québec, soit un écart de 4,7 \%.
La structure industrielle de la région était assez semblable à celle du Québec, bien que les secteurs primaire et secondaire y jouaient un rôle plus important. En 2013, le secteur primaire de la région représentait une proportion des emplois deux fois supérieure $(4,1 \%)$ à celle du Québec $(2,1 \%)$. La part des emplois dans le secteur de la fabrication était légèrement supérieure à celle du Québec (13,0 \% comparativement à $12,1 \%)$. Les principales activités de fabrication étaient axées sur la transformation des ressources naturelles. Le secteur tertiaire dominait en 2013 , avec $76,2 \%$ de la totalité des emplois en comparaison avec $79,4 \%$ pour le Québec. Dans la région, il était principalement tourné vers les services aux ménages et les services publics avec un déficit pour les services en lien avec l'économie du savoir.

L'indice de développement économique qui cerne la réalité économique des régions en tenant compte de la démographie, du marché du travail, du revenu et de la scolarité était estimé à 95,7 \% en 2012.

Habitée au départ par les Montagnais et exploitée par les Européens pour le commerce des fourrures aux dix-septième et dix-huitième siècles, la région ne fut ouverte à la colonisation qu'au cours du dix-neuvième siècle. L'installation de scieries et l'exploitation du bois pour la pulpe, puis le papier permirent le développement de la région au début du vingtième siècle. À partir des années 1920, les principaux cours d'eau furent harnachés pour la production électrique et l'implantation d'alumineries. Celles-ci stimuleront la croissance démographique et économique et le développement d'un marché local en croissance.

Les capitaux et les technologies utilisés pour l'exploitation des ressources provenaient de l'extérieur de la région et du pays et les produits étaient exportés à l'extérieur, principalement aux États-Unis.

Habitée au départ par les Montagnais et exploitée par les Européens pour le commerce des fourrures aux dix-septième et dix-huitième siècles, la région ne fut ouverte à la colonisation qu'au cours du dix-neuvième siècle. 


\section{Les limites d'utilisation des ressources} disponibles étant atteintes, les gains de productivité, produits par une modernisation accélérée des entreprises sous la pression de la mondialisation et des contraintes environnementales, ont conduit à une diminution de l'emploi dans ces secteurs d'activités.

Les ressources naturelles à la base du développement du Saguenay-Lac-Saint-Jean sont des ressources renouvelables. Elles ont permis la poursuite de la croissance économique et démographique de la région jusqu'à ce que leur plein potentiel soit exploité. Entre 1901 et 1981, la population est passée de 37367 à 285 284, soit une multiplication par 7. Par la suite, nous observons une légère baisse de la population qui est de 279863 en 2014.

Les limites d'utilisation des ressources disponibles étant atteintes, les gains de productivité, produits par une modernisation accélérée des entreprises sous la pression de la mondialisation et des contraintes environnementales, ont conduit à une diminution de l'emploi dans ces secteurs d'activités.

La modernisation des entreprises a permis d'améliorer les conditions de salaire des employés, d'améliorer la santé et la sécurité au travail, d'améliorer la qualité de l'air. Elle a également permis une utilisation plus efficace des ressources naturelles. Comme les gains de productivité sur la main-d'œuvre ont été plus grands que les gains de productivité sur l'utilisation des ressources naturelles, la modernisation des entreprises a été destructrice d'emplois.

Une étude que nous avons réalisée dans le secteur de l'aluminium de la région ${ }^{7}$ nous permet de mesurer l'ampleur de ce phénomène. De 1980 à 2003, un programme d'investissement de 7 milliards de dollars canadiens a été réalisé dans ce secteur.

La production en tonnes métriques a augmenté de $91 \%$. La production par travailleur a augmenté de $189 \%$. Le nombre d'emplois directs est passé de 9270 à 6115 soit une diminution de $34 \%$, mais le salaire moyen des travailleurs a augmenté de $68 \%$ en dollars constants. Les nouveaux procédés de production sont moins énergivores de $27 \%$, mais la consommation d'énergie par travailleur augmente de $119 \%$ compte tenu du fait que chaque travailleur produit davantage. Dans le secteur des pâtes et papiers, l'emploi direct passe de 4900 en 1981 à 1924 en 2006, soit une baisse de $60.7 \%$.

Une mobilisation de la communauté régionale dans le cadre du Sommet de développement régional de 1984 et également dans les mobilisations subséquentes ${ }^{8}$ a permis de cibler de nouveaux enjeux du développement comme la création d'un réseau de PME à forte croissance, le développement du secteur touristique, le développement du secteur tertiaire et de l'économie du savoir, la prise en compte des valeurs liées au respect de l'environnement et l'importance de se donner des pouvoirs accrus pour une plus grande maîtrise de son développement.

Ces efforts ont permis de créer de nouveaux emplois de sorte que la création nette d'emplois a augmenté au cours des dix dernières années, mais à un rythme moins grand que la moyenne du Québec. Ce déséquilibre au niveau de la croissance des emplois a entrainé une décroissance démographique avec un impact négatif sur la dynamique du marché local et par effet d'entrainement sur la dynamique économique et démographique.

Pour mesurer l'ampleur de ce phénomène, nous avons analysé la dynamique du marché du travail entre 2002 et $2012^{9}$.

Durant cette période, il y a une augmentation nette de 8800 emplois dans la région, soit de $7.5 \%$ en comparaison d'une augmentation de $11.8 \%$ pour l'ensemble du Québec, ce qui indique une sousperformance de création de 5029 emplois pour la région. La population de 15 ans et plus, la population en âge de travailler et de créer de la richesse, diminue de 1290 personnes dans la région soit une baisse de $0,06 \%$ alors qu'elle augmente de $10.8 \%$ dans l'ensemble du Québec, ce qui donne une sous-performance démographique de la région de 13819 personnes. Cette sous-performance démographique diminue, sans combler les écarts par rapport à la moyenne québécoise pour le taux d'emploi et les autres indicateurs du marché du travail. Le taux d'emploi, qui mesure la capacité 
d'une économie ou d'une région à fournir de l'emploi à sa population en âge de travailler, est de $55.9 \%$ par rapport à $60,0 \%$, ce qui indique une sous-performance régionale de 9240 emplois.

Entre 2002 et 2012, la région a créé 8800 emplois; il aurait fallu une augmentation supplémentaire de 24704 emplois pour permettre à la région d'offrir les mêmes opportunités d'emplois à une population en âge de travailler qui aurait augmenté au même rythme que la moyenne québécoise.

Pour établir un lien entre la dynamique économique et la dynamique démographique, nous avons mesuré le nombre d'emplois qu'il aurait fallu créer dans la région pour maintenir le même dynamisme démographique que le Québec et en même temps offrir les mêmes opportunités d'emplois. Nous obtenons ainsi une sous-performance économique de 24704 emplois.

\section{DEUXIÈME PARTIE}

\subsection{La contribution du projet à l'amélioration de l'efficacité économique}

\section{Les dépenses d'immobilisation ont généré pour les travailleurs de la région des revenus directs, indirects et induits de \\ 7,08 MS et la création de 140,5 emplois à temps complet durant la période de construction.}

Notre projet contribue à l'amélioration de l'efficacité économique du territoire par la création d'emplois, son impact positif sur l'attractivité du territoire et sa contribution à son positionnement stratégique sur une trajectoire d'avenir.

\section{La création d'emplois}

Notre projet a d'abord un impact positif par les emplois qu'il crée et aussi par ses effets d'entrainement sur les autres activités de la région.

Les dépenses d'immobilisation ont généré pour les travailleurs de la région des revenus directs, indirects et induits de 7,08 M\$ et la création de
Entre 2002 et 2012, la région a créé 8800 emplois; il aurait fallu une augmentation supplémentaire de 24704 emplois pour permettre à la région d'offrir les mêmes opportunités d'emplois à une population en âge de travailler qui aurait augmenté au même rythme que la moyenne québécoise.

Nous assistons à la naissance d'un cercle vicieux de sous-développement qui doit être brisé pour éviter le déclin à long terme de la région. Dans une perspective de développement territorial durable, il est d'autant plus nécessaire d'ouvrir de nouvelles voies stratégiques de développement que le respect de nouvelles normes pour la protection de l'environnement risque de provoquer la fermeture d'une usine donnant de l'emploi à 900 personnes dans le secteur de l'aluminium et que des mesures qui sont en discussion pour protéger le caribou forestier et augmenter les espaces protégés en forêt provoqueraient une perte importante d'emplois dans le secteur forestier.

140,5 emplois à temps complet durant la période de construction.

Les dépenses de fonctionnement en lien avec le programme de formation médicale et la présence des étudiants dans la région sont de $8 \mathrm{M} \$$ sur une base annuelle, soit des dépenses de 5,88 $\mathrm{M} \$$ pour les salaires et de 2,12 M\$ pour les achats de biens et services. Ces dépenses ont été calculées en utilisant 2011-2012 comme année de référence.

Ces dépenses de fonctionnement permettent à l'économie régionale d'enregistrer une augmentation annuelle récurrente des revenus pour les travailleurs de la région de $8,06 \mathrm{M} \$$. Les revenus directs sont de 5,88 M\$, les revenus indirects sont de 0,42 M\$ et les revenus induits sont de $1,76 \mathrm{M} \$$.

Elles permettent également de créer sur une base annuelle 148,2 emplois récurrents mesurés en équivalant à temps complet pour les travailleurs de la région. Parmi ceux-ci : 93,7 emplois sont des emplois directs, 10,5 sont des emplois indirects et 44 sont des emplois induits. 
Le projet contribue à améliorer l'image de la région et à la rendre plus attractive pour d'autres entreprises. La qualité de vie est un facteur important pour attirer une main-d'œuvre qualifiée et aussi retenir une population à la retraite qui est de plus en plus mobile.

Ces emplois sont des emplois de qualité, avec une rémunération annuelle moyenne supérieure de $35,6 \%$ à la moyenne régionale en 2012 , soit 54453 \$ en comparaison de $40144 \$$.

Selon les acteurs consultés dans notre enquête socio-économique, le programme aura également des impacts futurs importants sur la création d'emplois par sa contribution au recrutement de nouveaux médecins dans la région. Nous avons mesuré que sa contribution positive au recrutement de 63 nouveaux médecins spécialistes qui est prévu d'ici 2015 aurait pour effet, en plus des emplois et des revenus des spécialistes, de générer des revenus supplémentaires de 5,7 M\$ pour les travailleurs de la région et la création de 143 emplois, avec un salaire moyen de 40143 \$.

Selon la même enquête, le projet aura également des effets d'entrainement positifs sur la création dans la région de nouveaux programmes de formation dans le domaine de la santé et sur l'augmentation des activités de recherche qui sont très importantes dans ce domaine. Selon plusieurs répondants, des efforts importants doivent être faits pour développer de nouvelles activités de recherche en lien avec le programme.

Une estimation précise des emplois futurs n'a pas été faite, mais le potentiel de création de nouveaux emplois dans le secteur de la santé est important.

\section{L'attractivité du territoire}

Selon notre enquête, le programme est perçu comme un avantage comparatif important qui améliore le positionnement, le rayonnement et la réputation du Saguenay-Lac-Saint-Jean, tout en étant une source de fierté.

Le projet contribue à améliorer l'image de la région et à la rendre plus attractive pour d'autres entreprises. La qualité de vie est un facteur important pour attirer une main-d'œuvre qualifiée et aussi retenir une population à la retraite qui est de plus en plus mobile. L'accès à des soins de santé de qualité est un facteur important de la qualité de vie et par conséquent de l'attractivité de la région.

\section{Le repositionnement stratégique}

Le projet contribue également au repositionnement stratégique de la région sur une trajectoire d'avenir. Le secteur des services et plus particulièrement des services spécialisés est très important dans les pays développés à la fois pour les emplois créés et pour sa contribution à l'innovation. Le développement des activités de formation et de recherche dans le secteur de la santé permet de mieux positionner la région dans ce secteur d'activités.

\subsection{La contribution du projet à l'amélioration de l'équité sociale}

Le PFMS répond à un besoin prioritaire des habitants du territoire soit l'amélioration de l'accessibilité et de la qualité des soins en santé.

\section{L'accès aux soins}

La disponibilité des ressources est l'un des facteurs qui influencent l'accès aux soins ${ }^{10}$. Dans tous les territoires hors des grands centres du Québec et particulièrement dans les milieux ruraux, l'accessibilité aux soins en santé est déficiente en raison des difficultés de recruter suffisamment de médecins et de spécialistes pour répondre aux besoins de la population. Malgré les efforts et les incitatifs qui ont été mis en place par le gouvernement du Québec, le ratio médecin per capita y est plus faible que la moyenne québécoise.

Selon notre enquête, le PFMS facilite d'abord le recrutement d'une partie des étudiants qui sont formés dans la région. La connaissance de la région et la qualité de vie que l'on y trouve; des liens tissés avec les partenaires du réseau de la santé, une formation mieux adaptée aux besoins de la pratique médicale en région et des réseaux d'amis, sont des facteurs qui expliquent cette situation. Le PFMS a également un impact positif sur le recrutement dans les autres territoires périphériques du Québec. 
Le PFMS rend également le territoire plus attractif pour d'autres médecins compte tenu des opportunités qu'il leur offre de contribuer à des activités de formation universitaires et compte tenu aussi du dynamisme et de l'image positive qu'il crée dans le milieu médical.

Le PFMS rend également le territoire plus attractif pour d'autres médecins compte tenu des opportunités qu'il leur offre de contribuer à des activités de formation universitaires et compte tenu aussi du dynamisme et de l'image positive qu'il crée dans le milieu médical.

Selon la direction responsable du recrutement des médecins dans la région, les efforts de recrutement donnent de meilleurs résultats et l'accessibilité aux soins de santé s'est améliorée.

Le recrutement dans les régions rurales du territoire est encore difficile et il est souhaité que le PFMS établisse des liens plus étroits avec ces milieux.

Compte tenu du temps des médecins consacré à la formation, le temps disponible pour les soins de santé n'augmente que dans la mesure où le nombre des médecins augmente de sorte que ce sont les générations futures qui vont profiter pleinement des effets du programme sur l'accessibilité aux soins de santé.

\section{La qualité des soins}

Le PFMS permet d'améliorer la qualité des soins par une meilleure adaptation de la formation aux besoins de la pratique en région. Le PFMS doit certes donner une formation qui réponde aux standards de la formation nord-américaine du fait que les étudiants doivent réussir les mêmes examens nationaux pour obtenir leur droit de pratique. Les activités de formation pratique en région mettent cependant les étudiants en contact avec des problèmes plus variés et des clientèles plus diversifiées que dans les grands centres. Elles développent également une capacité d'autonomie plus grande chez les étudiants de sorte qu'ils peuvent mieux répondre aux besoins de la population.
Certains commentaires de notre enquête souhaitent que le programme se donne une couleur plus spécifique soit en lien avec des maladies héréditaires de la région ou encore avec une clientèle plus spécifique comme les Amérindiens.

Le PFMS permet également d'améliorer la qualité des soins dans le territoire par la dynamique de mise à jour des connaissances et de la recherche qu'il crée dans la communauté médicale. Les médecins qui enseignent ou encadrent des étudiants en stage ont de fortes incitations à mettre à jour leurs connaissances, ce qui provoque un effet d'entrainement positif chez leurs collègues. Ceux qui dirigent des activités de recherche mettent à jour des connaissances nouvelles qui permettront d'améliorer la qualité des soins pour la population régionale et aussi pour une population plus large.

Le PFMS apporte une contribution à la satisfaction d'un besoin de toute la population, sans référence à un groupe défavorisé particulier. Cependant, au niveau infraterritorial, il apporte une contribution à l'amélioration des revenus et des services à une région dont le revenu est inférieur à la moyenne québécoise. Dans ce sens, il contribue à une répartition plus équitable du développement au niveau national. De plus, comme le PFMS favorise l'accès aux études en médecine pour les étudiants de la région en diminuant les coûts, on peut penser que le programme profite davantage aux familles défavorisées pour l'accès de leurs enfants aux études en médecine.

\section{Le PFMS permet également d'améliorer la qualité des soins dans le territoire par la dynamique de mise à jour des connaissances et de la recherche qu'il crée dans la communauté médicale.}

\subsection{La contribution du projet au développement de la mobilisation des acteurs et de la gouvernance}

Le projet est d'abord le résultat de l'initiative et de la collaboration des acteurs régionaux. Comme le projet est une innovation dans le secteur complexe, la collaboration et l'appui d'un partenaire externe ont été nécessaires. De plus, l'appui du gouvernement du Québec a été essentiel. 
Le PFMS est le résultat d'une forte mobilisation de tous les acteurs régionaux. La population régionale, par la voix de ses élus, a donné son appui au programme, ce qui lui a donné sa légitimité politique.

Le PFMS est le résultat d'une forte mobilisation de tous les acteurs régionaux. La population régionale, par la voix de ses élus, a donné son appui au programme, ce qui lui a donné sa légitimité politique. L'hôpital régional (CSSSC) et l'université régionale (UQAC) ainsi que leurs dirigeants ont été des acteurs dont la collaboration a été essentielle. Sans la mobilisation des médecins de la région pour donner des cours et encadrer les stages des étudiants, le PFMS n'aurait pas été possible.

La région ne disposait pas de toute l'expertise nécessaire pour la création de ce programme et la contribution d'un partenaire externe était nécessaire. Une étude sur les innovations dans les petites et moyennes entreprises au Québec en arrive au même constat ${ }^{11}$. Le PFMS a nécessité la collaboration de l'Université de Sherbrooke qui avait établi des rapports de confiance de longue date avec le CSSSC et l'UQAC. Le PFMS est un programme délocalisé de l'Université de Sherbrooke. Il a été nécessaire de convenir des conditions de gestion du programme permettant de définir la collaboration de chaque partenaire en cherchant à maximiser l'autonomie pour les partenaires de la région. Pour faciliter cette collaboration, le premier doyen choisi pour diriger le programme a été un cadre responsable de la formation médicale au CSSSC et il a occupé un poste de doyen à la fois à l'Université de Sherbrooke et à l'UQAC.

Le PFMS a exigé la collaboration étroite de deux ministères du gouvernement du Québec, le ministère de la Santé et le ministère de l'Éducation. Avec l'appui des représentants de ces ministères en région, il a été possible de piloter le projet pour définir un projet permettant d'accueillir la contribution complémentaire de chacun de ces ministères. L'expertise en pilotage de projet disponible dans la région a été une condition essentielle à la réussite du projet, ce qu'on a appelé l'ingénierie territoriale ${ }^{1}$.
De plus, le projet a reçu plus facilement l'appui du gouvernement du Québec du fait qu'il avait comme objectif d'augmenter le nombre d'étudiants en médecine et qu'il était difficile de le faire dans les facultés existantes compte tenu des difficultés d'organiser des stages de formation dans les grands centres.

Le projet est le résultat de l'initiative, du dynamisme et de la collaboration des acteurs régionaux; il a cependant nécessité la contribution d'un partenaire externe et du gouvernement du Québec. Il est le résultat d'une collaboration interterritoriale.

\subsection{La contribution du projet au maintien de l'intégrité de l'environnement et du patrimoine collectif}

La capacité des générations futures de satisfaire leurs besoins est fortement liée au patrimoine collectif dont elles disposent ${ }^{12}$.

Sans impact négatif sur le capital naturel, le projet a permis d'améliorer, le capital d'infrastructure, le capital humain, le capital stratégique et le capital social qui font partie du patrimoine pour les générations futures.

Le projet n'apporte pas de contribution à la réduction du déficit environnemental régional et planétaire, mais les efforts appropriés ont été faits pour en minimiser les impacts environnementaux et maintenir en état le capital naturel.

Le PFMS est un projet dans le secteur des services et dans ce sens, son impact sur l'équilibre de l'écosystème régional est relativement faible. Le rapport Bruntland $^{13}$ recommandait d'ailleurs d'orienter l'économie des pays développés vers le secteur des services pour en diminuer l'impact écologique.

Les mesures ont été prises pour maximiser l'utilisation des ressources existantes au niveau de l'UQAC et du CSSSC et pour faciliter la communication à distance avec un équipement audiovisuel adapté.

En rapprochant les services de la population, le PFMS diminue les déplacements de la population régionale pour l'accès aux soins de santé et par conséquent, les émissions de $\mathrm{CO}_{2}$ liées à ces soins. 
Par ses dépenses d'immobilisation de 11,41 M\$, le PFMS a permis d'améliorer le capital d'infrastructure du territoire pour les services de formation et de santé. Les principales améliorations à l'UQAC sont la construction du pavillon de la médecine, de la passerelle et l'acquisition de matériel informatique. Les principales améliorations au CSSSC, quant à elles, sont des rénovations de salles et de cliniques pour recevoir les étudiants.

Le PFMS améliore également le capital humain en développant une expertise nouvelle dans le domaine de la formation. Les nombreux médecins qui participent aux activités de formation améliorent leurs connaissances et développent des connaissances nouvelles. Le recrutement de nouveaux médecins renforce également l'expertise qui est nécessaire pour des soins de qualité.

Par la nature des activités dont il favorise l'émergence, le PFMS améliore aussi le capital stratégique de la région et sa capacité d'innover et de créer des activités dans un secteur d'avenir.

Il apporte également une contribution significative au développement du capital social du territoire.

\section{CONCLUSION}

Dans la perspective du développement territorial durable, la promotion d'une plus grande efficacité économique, dans le respect des principes du développement durable, apparaît comme nécessaire pour permettre aux territoires en difficultés de sortir de leur dynamique de sous-développement.

L'analyse, à partir de la perception des acteurs sociaux, de la contribution d'un projet au développement d'un territoire en difficulté permet de proposer quelques pistes de réflexion pour enrichir le concept et la mise en œuvre d'une stratégie de développement territorial durable à l'échelle infranationale.

Dans la perspective du développement territorial durable, la promotion d'une plus grande efficacité économique, dans le respect des principes du développement durable, apparaît comme nécessaire pour permettre aux territoires
Le PFMS est le résultat d'une démarche complexe à la fois de mobilisation des acteurs régionaux et de l'organisation de la collaboration avec des acteurs externes au territoire. Sa réussite a permis de développer une expertise nouvelle dans ce domaine. Plusieurs répondants ont vanté les mérites de cette collaboration et ont fait des recommandations pour poursuivre cette collaboration dans d'autres domaines des soins de santé, en souhaitant une plus forte implication des milieux sociaux.

Le projet contribue à la satisfaction des besoins des générations présentes en permettant d'améliorer le patrimoine collectif qui sera à la disposition des générations futures.

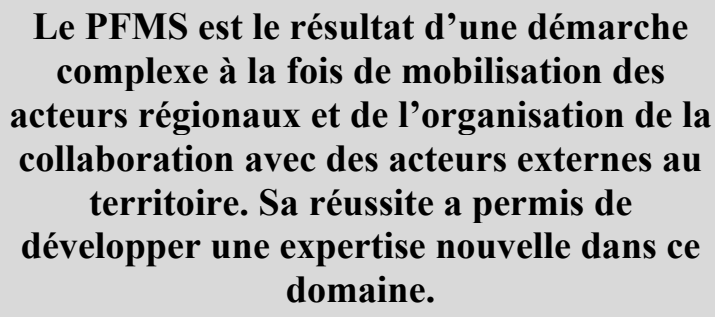

en difficultés de sortir de leur dynamique de sous-développement. Le maintien du dynamisme économique et du dynamisme démographique est nécessaire pour assurer la durabilité et la résilience des territoires. Les territoires ruraux et les territoires dont le développement repose sur l'exploitation des ressources naturelles sont particulièrement fragilisés dans le contexte actuel et leurs projets de développement devraient recevoir une attention particulière de la part des gouvernements nationaux.

Les projets et les stratégies peuvent contribuer à créer des emplois de qualité et améliorer l'attractivité des territoires. Dans les régions en difficultés, un repositionnement stratégique sur des trajectoires d'avenir est souvent nécessaire et les projets et les stratégies doivent être pensés et évalués dans cette perspective. Dans un environnement en changement rapide, la capacité d'innover est une condition gagnante. 
Dans une perspective de développement territorial durable, le développement est un processus d'apprentissage complexe et la mobilisation et la collaboration des acteurs du territoire est une condition essentielle.

Dans une perspective de développement territorial durable, la promotion de l'équité passe non seulement par une meilleure répartition des revenus, mais elle implique aussi l'utilisation des ressources pour répondre aux besoins prioritaires des citoyens et l'équité de la répartition géographique des services publics qui contribuent à l'amélioration du cadre de vie des citoyens. Un certain équilibre du développement sur le territoire national est souhaitable.

Certains territoires doivent passer progressivement d'une économie qui produit pour satisfaire les besoins externes du territoire à une économie qui vise à combler les besoins internes. L'organisation de la production de biens publics à proximité des citoyens qui les utilisent peut contribuer de façon significative à la mise en œuvre de cette stratégie.

Dans une perspective de développement territorial durable, le développement est un processus d'apprentissage complexe et la mobilisation et la collaboration des acteurs du territoire est une condition essentielle. Comme l'innovation est une condition nécessaire pour une stratégie de repositionnement stratégique des territoires en difficultés et que les ressources nécessaires ne sont pas toujours présentes dans le territoire, la collaboration de partenaires externes et du gouvernement national sont parfois nécessaires. Aux modèles de développement par le haut qui mettent l'accent sur la contribution des acteurs externes au territoire et aux modèles de développement par le bas qui mettent l'accent sur les acteurs internes, le développement territorial durable fait appel à un modèle de collaboration interterritoriale.

Dans une perspective de développement territorial durable, le développement repose sur l'exploitation et la mise en valeur du patrimoine du territoire et il permet d'enrichir ce patrimoine pour répondre aux besoins des générations futures. Alors que le développement durable accorde une importance toute particulière au capital naturel, le développement territorial accorde de l'importance à l'ensemble des composantes qui constitue le patrimoine d'un territoire. Il accorde une importance toute particulière au capital stratégique (le positionnement sur une trajectoire d'avenir), au capital humain (les compétences disponibles sur le territoire), au capital social (la capacité de mobiliser et de coordonner la contribution des acteurs sur le territoire). Le patrimoine prend des formes variées et parfois difficilement mesurables de sorte qu'il est difficile de mesurer l'apport net d'un projet au patrimoine du territoire. Des efforts de mesure sont nécessaires.

Suite à ces réflexions, dans la perspective des territoires infranationaux, le développement territorial durable pourrait se définir comme un développement qui utilise et met en valeur le patrimoine d'un territoire pour satisfaire les besoins prioritaires de la génération présente et qui enrichit ce patrimoine pour permettre aux générations futures de satisfaire leurs besoins; un développement qui permet de maintenir le dynamisme économique et démographique du territoire et de le positionner sur une trajectoire de développement d'avenir dans le respect des contraintes environnementales et des limites de la planète; un développement qui mobilise et organise la collaboration des partenaires locaux avec l'appui, si nécessaire, de partenaires externes et du gouvernement national.

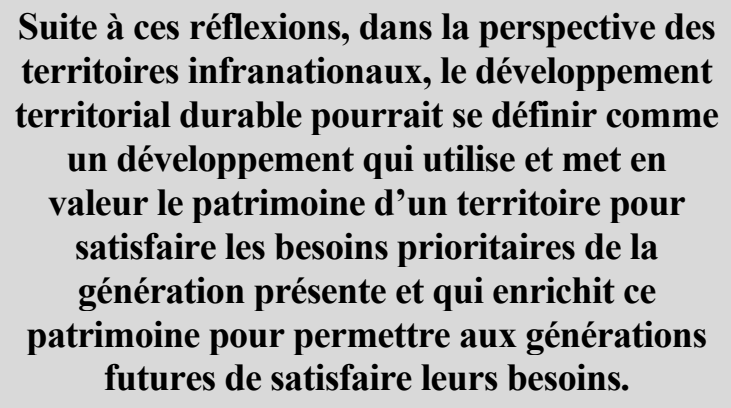




\section{BIBLIOGRAPHIE}

${ }^{1}$ Carrière J. P., Demazière C., Filimon L., Petrea R., 2013. La mise en ouvre du développement territorial durable : déclinaisons franco-roumaines, Éditions L'Harmattan.

${ }^{2}$ Bergeron, G. (sous la direction de), 2013. Les impacts économiques et sociaux de l'implantation d'un programme de formation médicale en région, le cas $d u$ programme de formation médicale à Saguenay, rapport de recherche, 88 pages, Colloque sur la formation de médecins en région, expériences comparables Saguenay, http://colloques.uqac.ca/medecineregion/ ${ }^{3}$ Ministère de l'Économie, de l'Innovation et des Exportations, été 2014. Saguenay-Lac-St-Jean, portrait régional.

${ }^{4}$ Angeon V., Caron P., Lardon S. Des liens sociaux à la construction d'un développement territorial durable: quel rôle de la proximité dans ce processus?, Développement durable et territoires [En ligne], Dossier 7 | 2006, mis en ligne le 10 mai 2006. URL : http://developpementdurable.revues.org/2851; DOI : 10.4000/developpementdurable.2851. Parra, C., Moulaert, F. La nature de la durabilité sociale : vers une lecture socioculturelle $d u$ développement territorial durable, Développement durable et territoires [En ligne], Vol. 2, n 2 | Mai 2011, mis en ligne le 04 mai 2011. URL : http://developpementdurable.revues.org/ 8970; DOI : 10.4000/develop-pementdurable.8970. Pecqueur B., avril 2012. La ressource territoriale: construit social et gouvernance spécifique, Séminaire Économies \& Territoires du GRIDEQ/CRDT, UQAR. Pecqueur B., septembre 2013. Postfordisme, territoire et durabilité, les nouveaux enjeux des politiques de projet territorial, dans Carrière J. P., Demazière C. Filimon L., Petrea R., La mise en œuvre du développement territorial durable : déclinaisons francoroumaines, Éditions L'Harmattan. Sustainable Development Solutions Network, mai 2014. Programme d'Actions pour le Développement Durable, rapport destiné au secrétaire général des Nations Unies. Theys J., 2002. La Gouvernance, entre innovation et impuissance [En ligne], Dossier 2 | 2002, mis en ligne le 01 novembre 2002. URL : http://developpement durablerevues.org/1523; DOI : 10.4000/developpement durable.1523. Villeneuve, C. et Riffon, O., 2011. 32 questions pour une réflexion plus large sur le développement durable, Département des Sciences Fondamentales, Université du Québec à Chicoutimi, http://ecoconseil.uqac.ca/.

${ }^{5}$ Demazière C. L'action économique locale et l'environnement. Les collectivités locales prennent-elles en compte les contraintes et opportunités qu'offrent les ressources naturelles pour l'économie d'un territoire? Développement durable et territoires [En ligne], Dossier 1 | 2002, mis en ligne le 01 septembre 2002. URL : http://developpementdurable.revues.org/894; DOI : 10.4000/develop-pementdurable.894. Centre for Rural and Northern Health Research, Lakehead University and Laurentian University, 2009. Exploring the SocioEconomic Impact of the Northern Ontario School of Medicine, Final Report.

${ }^{6}$ Laganier, R., Villalba B., et Zuindeau. B. Le développement durable face au territoire : éléments pour une recherche pluridisciplinaires, Développement durable et territoires [En ligne], Dossier 1 | 2002, mis en ligne le 01 septembre 2002. URL : http://developpement durable.revues.org/774; DOI : 10.4000/ developpement durable. 774 .

${ }^{7}$ Bergeron, G., printemps-été 2005. Analyse des avantages et des coûts de la politique de bas prix de l'énergie hydroélectrique : le cas d'Alcan au SaguenayLac-Saint-Jean, Revue Organisation et territoires.

${ }^{8}$ Bergeron, G., printemps-été 2004. De la reconversion industrielle à l'économie du savoir, Revue Organisations et territoires.

${ }^{9}$ Bergeron, G., 2002-2012. Une décennie décevante pour le développement économique et la création d'emplois dans la région du Saguenay-Lac-Saint-Jean, Cercle de presse de Saguenay, 2013.

${ }^{10}$ Touati N., Turgeon J. 2013. 14 Répartition géographique des médecins de famille : quelles solutions à un problème complexe. S.F.S.P.I, Santé Publique vol. 25 pages 465 à 473, http://www.cairn.info/revue-santepublique-2013-4-page-465.htm.

${ }^{11}$ Doloreux, D. et Melançon, Y., 2005. Réseaux d'innovation et compétivité dans les pme : quel rôle pour les territoires, $\mathrm{XLI}^{\mathrm{e}}$ colloque de l'ASRLDLF, Villes et territoires face aux défis de la mondialisation, Dijon.

${ }^{12}$ Gouvernement du Québec, Institut de la statistique du Québec et Ministère du Développement durable, de l'Environnement et de la Lutte contre les changements climatiques, juillet 2014, Recueil des indicateurs de suivi de la stratégie gouvernementale de développement durable 2008-2014. Jean, B., 2007. Du développement régional au développement territorial durable : vers un développement territorial solidaire pour réussir le développement des territoires ruraux, Coloquio international de Desenvovomento Territorial Sustentatavel, UFSC, Florianapolis, Brazil, 21 pages.

${ }^{13}$ Bruntland, G.H., 1988. Notre avenir à tous, Rapport de la commission sur l'environnement et le développement, Les Éditions du Fleuve. 